\title{
The effects of sequential presentation and spatial arrangements on the free classification of multidimensional stimuli'
}

\author{
STEPHEN HANDEL ${ }^{2}$ AND DAVID PREUSSER ${ }^{3}$ \\ KANSAS STATE UNIVERSITY
}

\begin{abstract}
Subjects classified sets of eight stimuli, constructed from three
\end{abstract} binary dimensions, into discrete groups in any way they wished. The stimuli were presented either simultaneously (all at once) or sequentially (one at a time). When stimuli were presented simultaneously, different spatial arrangements did not produce different classifications. When stimuli were presented sequentially, in some sequential orders the level of each dimension varied at a different position in the sequence. For these orders, Ss tended to use the two levels of the first dimension that varied to classify the stimuli into two groups. But, other orders in which the levels of all dimensions varied on the second stimulus did not influence Ss' classification. A comparison of sequential and simultaneous presentation showed for sequential presentation: (a) a smaller number of classifications was made using the dimensional structure to classify the stimuli, and $(b)$ of those classifications that were dimensional, fewer dimensions were used to classify the stimuli into groups.

In a free classification task, $\mathrm{S}$ sorts a set of multidimensional stimuli into discrete groups in any way he chooses. Each stimulus set typically represents the possible combinations of levels of two or more binary dimensions. The $\mathrm{S}$ usually selects one or two dimensions and places the stimuli into groups so that the different levels of these dimensions define the different groups (Imai, 1966). Two factors affecting the selection of dimensions have been found. First, $S$ is likely to select a dimension when he judges the levels of that dimension to be more dissimilar than the levels of other dimensions (Handel, 1967). Second, many Ss show distinct preferences for particular dimensions regardless of other factors (Imai \& Garner, 1965).

These studies have used the method of presenting all the stimuli simultaneously. Therefore, $\mathrm{S}$ was able to see the levels of all the dimensions before proceeding to classify the stimuli. However, stimuli may also be presented sequentially so that variation in the levels of dimensions may occur at different times. If so, some dimensions will become available for use in classification before other dimensions.

The purpose of this experiment was to compare simultaneous and sequential presentation of stimuli. Other purposes were to determine if (a) making dimensions available for classification at different points in the sequence influenced free classification, and (b) whether the spatial arrangement of the stimuli when presented simultaneously influenced classification.

\section{METHOD}

Stimuli

Three types of stimulus materials were used: black lines, purple colors, and colors distributed across the color space. For each stimulus set, all possible combinations of three binary dimensions were used, so that there were eight stimuli in each set. Within each type of material, different sets were constructed by using different levels of dimensions. The identical stimulus sets were used for simultaneous and sequential presentation.

Line stimuli. The line stimuli, drawn on $3 \times 5$ in. white cards,

\begin{tabular}{|c|c|c|c|}
\hline \multirow{2}{*}{$\begin{array}{c}\text { Stimulus } \\
\text { Set }\end{array}$} & \multicolumn{3}{|c|}{ Dimension } \\
\hline & Width (in.) & Length (in.) & Orientation (deg) \\
\hline 1 & $2 / 32,3 / 32$ & $12 / 8,16 / 8$ & 40,50 \\
\hline 2 & $2 / 32,3 / 32$ & $12 / 8,16 / 8$ & 35,55 \\
\hline 3 & $3 / 32,6 / 32$ & $11 / 8,17 / 8$ & 40,50 \\
\hline 4 & $2 / 32,6 / 32$ & $1 \quad 1 / 8,17 / 8$ & 30,60 \\
\hline 5 & $2 / 32,8 / 32$ & $1,24 / 8$ & 15,75 \\
\hline
\end{tabular}

were black lines that varied in length, width, and orientation. There were five different sets of line stimuli and the magnitudes of the levels of the dimensions are shown in Table 1 .

Purple colors. Colors varying in hue, value, and chroma were selected from the purple section of the Munsell glossy book. There were three different sets of purple colors and the magnitudes of the levels of the dimensions are shown in Table 2.

Each color chip was $1-3 / 8$ in. $x 1-5,8$ in and was mounted in the center of a $3 \times 5$ in. white card.

Distributed colors. The distributed colors ranged across the Munsell color space. The rationale for the use of these stmul is detailed in Handel (1967). The three dimensions werc the 5R-5BG and the 10Y-10PB lines in the Munsell sy stem and the Munsell value dimension Fach set consisted of four hues green, blue, yellow-red, purple each at wo levels of 'ighing i M nith value).

To describe the dimensional structure of these stimul further, if $S$ s use the 5R-5BG dimension for classification, the green and blue hues are placed in one group and the remaining hues are placed in the second group. If Ss use the 10Y-10PB dimension for classification, the green and yellow-red hues are placed in one group and the remaining hues are placed in the second group. If Ss use the value dimension for classification, the four lighter hues are placed in one group and the four darker hues are placed in the second group. If $\mathrm{Ss}$ use both the 5R-5BG and 10Y-10PB dimensions for classification, there are four groups defined by hue. Finally, Ss could use either the 5R-5BG and value dimensions or the 10Y-10PB and value dimensions to classify the stimuli into four groups. However, these two classifications were used rarely, only 2 of 270 classifications used either of these schemes.

There were three stimulus sets and the colors in each set are

Table 2

Purple Color Stimuli: Magnitudes of the Levels of Each Dimension for Every Stimulus Set.

\begin{tabular}{clcl}
\hline $\begin{array}{c}\text { Stimulus } \\
\text { Set }\end{array}$ & \multicolumn{3}{c}{ Dimension } \\
& \multicolumn{1}{c}{ Hue } & Value & Chroma \\
\hline 1 & $10 \mathrm{P}, 10 \mathrm{RP}$ & 3,6 & 4,8 \\
2 & 10P, 10RP & 4,5 & 4,8 \\
3 & $2.5 \mathrm{RP}, 7.5 \mathrm{RP}$ & 3,6 & 4,8 \\
\hline
\end{tabular}


Table 3

Distributed Color Stimuli: Hues and Chromas of Four Colors in Each Stimulus Set.

\begin{tabular}{|c|c|c|c|c|}
\hline \multirow{2}{*}{$\begin{array}{c}\text { Stimulus } \\
\text { Set }\end{array}$} & \multicolumn{4}{|c|}{ Color } \\
\hline & Green & Blue & Yellow-Red & Purple \\
\hline 1 & $2.5 \mathrm{G} / / 2$ & $7.5 B / / 2$ & $7.5 \mathrm{YR} / / 2$ & 2.5RP/ / 2 \\
\hline 2 & $2.5 G / / 4$ & $7.5 \mathrm{~B} / / 4$ & $7.5 \mathrm{YR} / / 4$ & $2.5 \mathrm{RP} / / 4$ \\
\hline 3 & $10 G Y / / 8$ & $10 B / / 8$ & $10 \mathrm{YR} / / 8$ & $10 \mathrm{P} / / 8$ \\
\hline
\end{tabular}

Note: The magnitudes of the value dimension are 4 and 5.

shown in Table 3 . The color chips were from the Munsell glossy book and were mounted in the same way as the purple colors.

\section{Simultaneous Presentation}

Subjects. The Ss were 30 Kansas State University undergraduates recruited from introductory psychology classes. The Ss were randomly assigned to conditions. Colorblind $S$ s were not used.

Spatial arrangements. The eight stimuli in a set were placed in front of $S$ in two columns of four stimuli apiece, so that one dimension could be used for classification by placing the stimuli in one column into one group and the stimuli in the second column into another group; a second dimension could be used for classification by placing the top two rows in one group and the bottom two rows in another group. The third dimension could be used for classification by placing the first and third rows in one group and the second and fourth rows in another group.

The six possible spatial arrangements that could be constructed by assigning one dimension to each of the three spatial positions were used. The choice of these particular arrangements was based on pilot studies. In these studies, the stimuli were presented in a wide variety of arrangements, including a completely random presentation. The six arrangements chosen seemed to influence which dimensions were chosen for use in classification. None of the arrangements affected the percentage of nondimensional (miscellaneous) classifications. Even with completely random presentation, only $5 \%$ of the two-group classsifications were nondimensional.

Procedure. The Ss first sorted the stimuli into any number of groups, in any way they wished. The only restrictions were that Ss could not place all the stimuli in one group or place each stimulus in a separate group. Then Ss were asked to place the stimuli into two groups in any way they wished. The Ss who had used two groups originally were free to change their classification.

Experimental design. Each $\mathbf{S}$ was presented all stimulus sets. Five of the spatial arrangements were used twice and one was used once. Different spatial arrangements were used for each set of one stimulus type. Each stimulus set by spatial arrangement combination was presented to five Ss. The order of presentation of the stimulus sets was counterbalanced.

\section{Sequential Presentation}

Subjects. The Ss were 60 different Kansas State University undergraduates recruited from introductory psychology classes. The Ss were randomly assigned to conditions. Colorblind Ss were not used.

Sequences of presentation. Two different sequences were used. In Sequence 1, the level of one dimension varied on the second stimulus, the level of another dimension varied on the third stimulus, and the level of the third dimension varied on the fifth stimulus. Therefore, each dimension became available for classification at a different position in the sequence. The three different orders of Sequence 1, shown in Table 4, alternate the position at which each dimension varied. Thus, differences in classifications among the three orders must be due to the position at which each dimension varied.
In Sequence 2, the level of all dimensions varied on the second stimulus. The third stimulus differed from the first in the level of one dimension in order to bias Ss against using this dimension for classification. The three orders of Sequence 2, to bias Ss against each dimension, are also shown in Table 4.

Procedure. The Ss were presented the stimulj one at a time, began to classify the stimuli after the third stimulus, and continued to classify each additional stimulus. The task was self-paced and Ss were free to change their classification at any point. After classifying the eight stimuli, Ss were asked to place the stimuli into two groups. Again, Ss who had originally placed the stimuli into two groups were free to change their classification.

Experimental design. Each S was presented all stimulus sets. Five of the orders of presentation were used twice, and the remaining order was used once. Different orders of presentation were used for each set of one stimulus type. Each stimulus set by order of presentation combination was presented to $10 \mathrm{Ss}$. The order of presentation of the stimulus sets was counterbalanced.

\section{Statistical Analysis}

The majority of statistical tests are chi-square contingency tests between the different spatial arrangements (or different sequential orders) and the distributions of classifications.

The contingency between the experimental conditions and the distribution of classifications for both simultaneous and sequential presentation can be measured at three points: the original classifications, the reclassifications into two groups, and the overall classifications into two groups. The overall classifications into two groups were essentially the summation of the original classifications into two groups and the reclassifications into two groups, since Ss rarely changed their original two-group classifications.

The original classifications were broken into seven categories: the three possible classifications into two groups using the levels of one dimension, the three possible classifications into four groups using the levels of two dimensions, and the remaining miscellaneous classifications.

When the stimuli were classified into two groups, there were four categories: the three possible classifications using the levels of one dimension, and the miscellaneous classifications.

The classifications of all sets of one type of stimulus material were combined for each analysis in order to have (a) representative sets of stimuli for each stimulus type, and (b) a fairly large expected value for the chi-square test. Two statistical objections

Table 4

Sequential Presentation: The Three Orders of Sequence 1 and Sequence 2.

\begin{tabular}{|c|c|c|c|c|c|c|c|c|c|c|}
\hline \multirow[t]{3}{*}{ Sequence } & \multirow{3}{*}{$\begin{array}{c}\text { Stimulus } \\
\text { Card }\end{array}$} & \multicolumn{9}{|c|}{ Order } \\
\hline & & \multicolumn{3}{|c|}{$\frac{1}{\text { Dimension }}$} & \multicolumn{3}{|c|}{$\begin{array}{c}2 \\
\text { Dimension }\end{array}$} & \multicolumn{3}{|c|}{$\stackrel{3}{\text { Dimension }}$} \\
\hline & & 1 & 2 & 3 & 1 & 2 & 3 & 1 & 2 & 3 \\
\hline \multirow{8}{*}{1} & 1 & 0 & 0 & 0 & 0 & 0 & 0 & 0 & 0 & 0 \\
\hline & 2 & 0 & 0 & 1 & 0 & 1 & 0 & 1 & 0 & 0 \\
\hline & 3 & 0 & 1 & 0 & 1 & 0 & 0 & 0 & 0 & 1 \\
\hline & 4 & 0 & 1 & 1 & 1 & 1 & 0 & 1 & 0 & 1 \\
\hline & 5 & 1 & 0 & 0 & 0 & 0 & 1 & 0 & 1 & 0 \\
\hline & 6 & 1 & 0 & 1 & 0 & 1 & 1 & 1 & 1 & 0 \\
\hline & 7 & 1 & 1 & 0 & 1 & 0 & 1 & 0 & 1 & 1 \\
\hline & 8 & 1 & 1 & 1 & 1 & 1 & 1 & 1 & 1 & 1 \\
\hline \multirow{8}{*}{2} & 1 & 0 & 0 & 0 & 0 & 0 & 0 & 0 & 0 & 0 \\
\hline & 2 & 1 & 1 & 1 & 1 & 1 & 1 & 1 & 1 & 1 \\
\hline & 3 & 0 & 0 & 1 & 0 & 1 & 0 & 1 & 0 & 0 \\
\hline & 4 & 1 & 1 & 0 & 1 & 0 & 1 & 0 & 1 & 1 \\
\hline & 5 & 0 & 1 & 0 & 1 & 0 & 0 & 0 & 0 & 1 \\
\hline & 6 & 1 & 0 & 1 & 0 & 1 & 1 & 1 & 1 & 0 \\
\hline & 7 & 0 & 1 & 1 & 1 & 1 & 0 & 1 & 0 & 1 \\
\hline & 8 & 1 & 0 & 0 & 0 & 0 & 1 & 0 & 1 & 0 \\
\hline
\end{tabular}


Table 5

The Percentages of Initial Classifications for each Type of Stimulus Material Using (a) the Levels of One Dimension to Classify the Stimuli into Two Groups, (b) the Levels of Two Dimensions to Classify the Stimuli into Four Groups, or (c) Miscellaneous Classifications.

Method of

Presentation
Type of Stimulus Material

\begin{tabular}{|c|c|c|c|c|c|c|c|c|c|}
\hline & \multicolumn{3}{|c|}{ Lines } & \multicolumn{3}{|c|}{$\begin{array}{c}\text { Purple } \\
\text { Colors }\end{array}$} & \multicolumn{3}{|c|}{$\begin{array}{l}\text { Distributed } \\
\text { Colors }\end{array}$} \\
\hline & $2 \mathrm{gps}$ & $4 \mathrm{gps}$ & Misc & $2 \mathrm{gps}$ & $4 \mathrm{gps}$ & Misc & $2 \mathrm{gps}$ & $4 \mathrm{gps}$ & Misc \\
\hline $\begin{array}{l}\text { Simultaneous } \\
\text { Sequence } 1 \\
\text { Sequence } 2\end{array}$ & $\begin{array}{l}37 \\
59 \\
51\end{array}$ & $\begin{array}{l}61 \\
29 \\
31\end{array}$ & $\begin{array}{r}2 \\
12 \\
18\end{array}$ & $\begin{array}{l}31 \\
42 \\
39\end{array}$ & $\begin{array}{l}60 \\
10 \\
23\end{array}$ & $\begin{array}{r}9 \\
48 \\
38\end{array}$ & $\begin{array}{l}27 \\
21 \\
22\end{array}$ & $\begin{array}{l}72 \\
61 \\
58\end{array}$ & $\begin{array}{r}1 \\
18 \\
20\end{array}$ \\
\hline
\end{tabular}

Note: The percentages are averaged across all stimulus sets for each type of stimulus material and across the six spatial arrangements for Simultaneous Presentation or the three orders for each Sequence.

can be raised to this procedure. The first objection is that some cells in the tables will still have small expected values, a situation that may give a spuriously high chi-square statistic for that cell. To guard against this possibility, the chi-square statistic was calculated separately for each cell in the contingency trble, and in none of the reported results do cells with small expected values contribute heavily to the overall chi-square value of the table. The second objection is that $S$ can appear once for all spatial arrangements or sequential orders so that the independence assumption is violated. However, $\mathrm{Ss}$ show preferences for dimensions (Imai \& Garner, 1965), and since the analyses attempt to show differences in classification as a result of the experimental conditions, the lack of independence should produce a conservative chi-square test.

\section{Simultaneous Presentation}

\section{RESULTS}

The different spatial arrangements did not influence the initial classifications for any type of stimulus material. The percentages of initial classifications using the levels of one or two dimensions to classify the stimuli into two or four groups and the percentage of miscellaneous classifications, averaged across all six spatial arrangements for each type of stimulus by material, are shown in Table 5. For all stimulus types, the majority of classifications used the levels of two dimensions to place the stimuli into four groups.

In addition, the spatial arrangements did not influence the choice of dimension used to classify the stimuli into two groups for any stimulus type. The percentages of times each dimension was used to classify each type of stimulus material into two groups, averaged across all arrangements, are shown in Table 6.

\section{Sequential Presentation}

Sequence 1. The percentages of initial classifications using the levels of one or two dimensions to classify the stimuli into two or four groups and the percentages of miscellaneous classifications, for each order of Sequence 1 and type of stimulus material, are shown in Table 5 .

The three orders of Sequence 1 influenced the initial classification of the line stimuli $\left(\chi^{2}(12)=30.8, p<.01\right)$ and the purple color stimuli $\left(\chi^{2}(12)=30.4, p<.01\right)$, but not the distributed color stimuli. For both the line and purple color stimuli, the majority of classifications used the levels of one dimension to split the stimuli into two groups and the orders influenced primarily which dimension was chosen. The dimension that varied on the second stimulus was used for $45 \%$ of the classifications, the dimension that varied on the third stimulus was used for $40 \%$ of the classifications, and the dimension that varied on the fifth stimulus was used for $15 \%$ of the classifications. The orders did not significantly influence which two dimensions were chosen to split the stimuli into four groups, or the percentage of two-group, four-group, or miscellaneous classifications.

By contrast, the majority of classifications of the distributed color stimuli were into four groups $(61 \%)$, using the $5 \mathrm{R}-5 \mathrm{BG}$ and $10 \mathrm{Y}-10 \mathrm{~PB}$ dimensions. Therefore, since the major effect of the orders was to influence the two group classifications, the three orders of Sequence 1 did not produce different classifications of the distributed colors.

When the stimuli were reclassified into two groups, the orders of Sequence 1 did not influence the two-group reclassifications of the line or purple color stimuli, but did influence which dimension was chosen to reclassify the distributed colors into two groups $\left(\chi^{2}(6)=13.3, \mathrm{p}<.05\right)$. The effect of the orders on dimensional choice for the distributed color stimuli was similar to that for the line and purple color stimuli. For the distributed colors, the dimension that varied on the second stimulus was used for $50 \%$ of the classifications, the dimension that varied on the third stimulus was used for $27 \%$ of the classifications, and the dimension that varied on the fifth stimulus was used for $23 \%$ of the classifications.

For each order of Sequence 1, the overall percentage of times each dimension was used to classify each type of stimulus material into two groups is shown in Table 6. The different orders of Sequence 1 produce different two-group classifications for the distributed colors $\left(\chi^{2}(4)=13.6, p<.01\right)$, for the purple colors $\left(\chi^{2}(6)=14.9, p<.025\right)$, and less strongly for the lines $\left(\chi^{2}(4)=8.0, p<.10\right) .4$ Overall, the effect of the position at which each dimension varied in the sequence was smaller $(38 \%$ classifications at the second stimulus, $33 \%$ classifications at the third stimulus, 23\% classifications at the fifth stimulus, and $7 \%$ miscellaneous classifications), but still significant (Friedman Two-way Analy sis of Variance, $\mathrm{p}<.01$ ).

Sequence 2. The three different orders of Sequence 2 did not influence the initial classifications of any stimulus type (shown in Table 5), the subsequent reclassifications into two groups, or the overall percentages of two-group classifications (shown in Table 6).

\section{Simultaneous Presentation}

\section{DISCUSSION}

The spatial arrangement of a set of stimuli did not influence

Table 6

The Overall Percentages of Times Each Dimension was Used to Classify Each Type of Stimulus Material into Two Groups.

\begin{tabular}{|c|c|c|c|c|c|c|}
\hline \multirow{4}{*}{$\begin{array}{l}\text { Type of } \\
\text { Stimulus } \\
\text { Material }\end{array}$} & \multirow[t]{4}{*}{ Dimension } & \multicolumn{5}{|c|}{ Method of Presentation } \\
\hline & & \multicolumn{4}{|c|}{ Sequential Presentation } & \multirow{3}{*}{$\begin{array}{l}\text { Simultaneous } \\
\text { Presentation }\end{array}$} \\
\hline & & \multicolumn{3}{|c|}{$\begin{array}{c}\text { Sequence } 1 \\
\text { Order }\end{array}$} & \multirow[t]{2}{*}{ Sequence 2} & \\
\hline & & 1 & 2 & 3 & & \\
\hline \multirow[t]{4}{*}{ Lines } & Width & 36 & 50 & 64 & 64 & 63 \\
\hline & Length & 28 & 26 & 16 & 13 & 30 \\
\hline & Orientation & 32 & 22 & 18 & 21 & 7 \\
\hline & Misc & 4 & 2 & 2 & 2 & 0 \\
\hline Purple & Hue & 10 & 13 & 20 & 13 & 11 \\
\hline \multirow[t]{3}{*}{ Colors } & Value & 60 & 57 & 27 & 42 & 47 \\
\hline & Chroma & 7 & 27 & 33 & 23 & 42 \\
\hline & Misc & 23 & 3 & 20 & 21 & 0 \\
\hline Distributed & $5 \mathrm{R}-5 \mathrm{BG}$ & 30 & 57 & 53 & 47 & 65 \\
\hline \multirow[t]{3}{*}{ Colors } & $10 \mathrm{Y}-10 \mathrm{~PB}$ & 7 & 17 & 7 & 4 & 5 \\
\hline & Value & 63 & 26 & 30 & 38 & 30 \\
\hline & Misc & 0 & 0 & 10 & 11 & 0 \\
\hline
\end{tabular}

Note: The percentages are averaged across all stimulus sets for each type of stimulus material and across the six spatial arrangements for Simultaneous Presentation or the three orders for Sequence 2. 
the classification of these stimuli. This result suggests that any effect of sequential presentation on classification is not the result of the spatial arrangement used by Ss when classifying the stimuli.

\section{Sequential Presentation}

Sequence 1 , in which dimensions varied at different stimuli in the sequence, influenced dimensional choice. For the line and purple color stimuli, the dimension that varied on the second stimulus was initially used for classification, and Ss often continued to use this dimension exclusively for classification. The Ss did not reorganize when other dimensions became available and infrequently used the initial dimension in combination with another dimension to classify the stimuli into more than two groups. For the distributed color stimuli, the initial classifications were not affected by order, but the subsequent reclassifications into two groups were affected by the order in which the dimensions varied. Therefore, for all stimulus materials, the orders of Sequence 1 only influenced which dimension was used to classify the stimuli into two groups.

However, Sequence 2, in which all dimensions vary simultaneously on the second stimulus, did not influence classification. Since all three dimensions varied on the second stimulus, Ss perceived the levels of all dimensions before proceeding to classify the remaining stimuli. Thus, once the possible dimensional variation is known, the order of the subsequent stimuli did not influence classification. This conclusion is supported and generalized by the results using simultaneous presentation; when $S$ is able to view all the stimuli at once, their arrangement does not influence classification. In summary, once $S$ knows the dimensional structure, neither sequential order nor spatial arrangement influences free classification. On the other hand, when stimuli are presented using Sequence $1, S$ uses the first dimension to vary for classification and becomes "blind" to the variation of the remaining two dimensions.

\section{Comparison Between Simultaneous and Sequential Presentation}

The major difference between simultaneous and sequential presentation was the number of groups used by Ss for their initial classification. When the stimuli were presented simultaneously, for all types of stimulus sets, the majority of the classifications (64\%) used the levels of two dimensions to classify the stimuli into four groups, and $31 \%$ of the classifications used the levels of one dimension to classify the stimuli into two groups. 5 However, using sequential presentation, the distributed color stimuli were the only stimulus type in which classification into four groups was the prevalent mode of classification. For the line and purple color stimuli, $48 \%$ of the classifications used the levels of one dimension to classify the stimuli into two groups, and $23 \%$ of the classifications used the levels of two dimensions to classify the stimuli into four groups.
A second difference between simultaneous and sequential presentation was that, in the initial classifications, there were more than six times as many miscellaneous classifications for sequential presentation (26\%) than when using simultaneous presentation ( $4 \%$ ). Furthermore, for the two-group classifications, when using sequential presentation, $10 \%$ of the classifications were miscellaneous, but when using simultaneous presentation no classifications were miscellaneous.

These results suggest that Ss are more aware of the dimensional structure of the stimuli when they are presented simultaneously; Ss use a greater number of dimensions to classify the stimuli into groups and there are fewer miscellaneous classifications. These results are not due simply to Ss not knowing the levels of the dimensions, since in Sequence 2, the levels of all the dimensions changed on the second stimulus. Moreover, these conclusions are not due to the particular spatial arrangements used, as preliminary work had shown that the percentage of miscellaneous classifications was independent of arrangements.

\section{REFERENCES}

HANDEL, S. Classification and similarity of multidimensional stimuli. Perceptual \& Motor Skills, 1967, 24, 1191-1203.

IMAI, S. Classification of sets of stimuli with different stimulus characteristics and numerical properties. Perception \& Psychophysics, 1966, 1, 48-54.

IMAI, S., \& GARNER, W. R. Discriminability and preference for attributes as factors in free and constrained classification. Journal of Experimental Psychology, 1965, 69, 596-608.

\section{NOTES}

1. This research was supported in part by the Bureau of General Research, Kansas State University, and in part by NASA University Sustaining Grant NsG-692. This work was carried out during the second author's tenure as an NDEA Fellow.

2. Address: Department of Psychology, Anderson Hall, Kansas State University, Manhattan, Kansas 66502.

3. Now at $Y$ ale University.

4. The chi-square test for the line and distributed color stimuli was a 3 by 3 contingency table (orders by dimensions) since only $3 \%$ of the classifications were miscellaneous.

5. For an independent comparison, the initial classifications using simultaneous presentation were tabulated from the data of Handel (1967). For line stimuli (the dimensions were length, orientation, and lateral position), $26 \%$ of the classifications used the levels of one dimension to place the stimuli into two groups, $69 \%$ of the classifications used the levels of two dimensions to place the stimuli into four groups, and $5 \%$ of the classifications were miscellaneous. For purple color stimuli (the dimensions were hue, value, and chroma), $24 \%$ of the classifications were into two groups, $57 \%$ of the classifications were into four groups, and $19 \%$ of the classifications were miscellaneous. For the identical sets of distributed color stimuli used in the present experiment, $13 \%$ of the classifications were into two groups, $74 \%$ of the classifications were into four groups, and $13 \%$ of the classifications were miscellaneous. Thus, in both studies, when using simultaneous presentation, the majority of the initial classifications used the levels of two dimensions to classify the stimuli into four groups.

(Accepted for publication February 19, 1969.) 